\title{
Article \\ A Hypomorphic Mutant of PHD Domain Protein Male Meiocytes Death 1
}

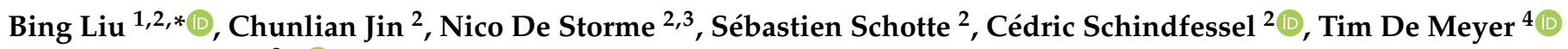 \\ and Danny Geelen $2, *$ (D) \\ 1 College of Life Sciences, South-Central University for Nationalities, Wuhan 430074, China \\ 2 Unit HortiCell, Department of Plants and Crops, Faculty of Bioscience Engineering, Ghent University, \\ 9000 Ghent, Belgium; chunlian.jin@ugent.be (C.J.); nico.destorme@kuleuven.be (N.D.S.); \\ Sebastien.Schotte@UGent.be (S.S.); Cédric.Schindfessel@UGent.be (C.S.) \\ 3 Division of Crop Biotechnics, Department of Biosystems, KU Leuven, 3001 Leuven, Belgium \\ 4 Department of Data Analysis and Mathematical Modelling, Ghent University, Coupure Links, \\ 9000 Ghent, Belgium; Tim.DeMeyer@UGent.be \\ * Correspondence: bl472@scuec.edu.cn (B.L.); Danny.Geelen@UGent.be (D.G.); Tel.: +86-1355-403-9531 (B.L.); \\ +32-(0)-9264-6076 (D.G.)
}

check for updates

Citation: Liu, B.; Jin, C.; De Storme, N.; Schotte, S.; Schindfessel, C.; De Meyer, T.; Geelen, D. A Hypomorphic Mutant of PHD Domain Protein Male Meiocytes Death 1. Genes 2021, 12, 516. https:// doi.org/10.3390/genes12040516

Academic Editor: Kejian Wang

Received: 9 March 2021

Accepted: 26 March 2021

Published: 1 April 2021

Publisher's Note: MDPI stays neutral with regard to jurisdictional claims in published maps and institutional affiliations.

Copyright: (C) 2021 by the authors. Licensee MDPI, Basel, Switzerland. This article is an open access article distributed under the terms and conditions of the Creative Commons Attribution (CC BY) license (https:/ / creativecommons.org/licenses/by/ $4.0 /)$.

\begin{abstract}
Meiosis drives reciprocal genetic exchanges and produces gametes with halved chromosome number, which is important for the genetic diversity, plant viability, and ploidy consistency of flowering plants. Alterations in chromosome dynamics and/or cytokinesis during meiosis may lead to meiotic restitution and the formation of unreduced microspores. In this study, we isolated an Arabidopsis mutant male meiotic restitution 1 ( $m m r 1$ ), which produces a small subpopulation of diploid or polyploid pollen grains. Cytological analysis revealed that $m m r 1$ produces dyads, triads, and monads indicative of male meiotic restitution. Both homologous chromosomes and sister chromatids in $m m r 1$ are separated normally, but chromosome condensation at metaphase I is slightly affected. The mmr 1 mutant displayed incomplete meiotic cytokinesis. Supportively, immunostaining of the microtubular cytoskeleton showed that the spindle organization at anaphase II and mini-phragmoplast formation at telophase II are aberrant. The causative mutation in mmr1 was mapped to chromosome 1 at the chromatin regulator Male Meiocyte Death 1 (MMD1/DUET) locus. mmr1 contains a C-to-T transition at the third exon of MMD1/DUET at the genomic position $2168 \mathrm{bp}$ from the start codon, which causes an amino acid change G618D that locates in the conserved PHD-finger domain of histone binding proteins. The F1 progenies of mmr1 crossing with knockout mmd1/duet mutant exhibited same meiotic defects and similar meiotic restitution rate as $m m r 1$. Taken together, we here report a hypomorphic $m m d 1$ / duet allele that typically shows defects in microtubule organization and cytokinesis.
\end{abstract}

Keywords: meiosis; meiotic restitution; meiotic cytokinesis; unreduced gametes; MMD1

\section{Introduction}

Most higher plants, especially for angiosperms, have undergone at least one round of whole genome duplication (WGD) in evolution [1,2]. Formation and fusion of diploid or polyploid gametes are considered the primary route to plant polyploidization [3,4]. Alterations in one or more meiosis processes, including omission of meiotic cell cycle, defective chromosome segregation, spindle misorientation, and/or incomplete meiotic cytokinesis, are the common mechanisms leading to the generation of unreduced gametes through restitution of meiotic cell division [5-10]. In concern of the fundamental significance and practical utilization for polyploid crop breeding, it is of particular importance to uncover the genetic factors involved in unreduced gamete formation.

In Arabidopsis, at least 23 genes have been identified, which, in case of dysfunction, cause meiotic restitution (Table 1). Typically, functional mutations of the genes that control 
meiotic cell cycle transition, such as OSD1/GIG1 and TAM/CYCA1;2, terminate the meiosis program prematurely, thereby inducing the production of $2 \mathrm{n}$ eggs and $2 \mathrm{n}$ spores [11]. Moreover, alterations of the three-dimensional positioning of spindles may result in unreduced gamete formation. The mechanism asserting the perpendicular position of the spindles is lost in parallel spindle1 (ps1) and jason mutants, which results in partial or complete fusion of the spindles [12,13]. Male meiotic cytokinesis in Arabidopsis is regulated by a mitogenactivated protein kinase (MAPK) signaling cascade composed of TES/STUD/AtNACK2ANPs-MKK6/ANQ-MPK4. Loss of function of any member in this module leads to defects in male meiotic cytokinesis and the formation of pollen grains with an increased ploidy level [14-18]. Remarkably, the chromatin regulator Male Meiocyte Death 1 (MMD1/DUET), a PHD-finger protein that reads and binds with H3K4methylation sites, plays an important role in regulating multiple processes in Arabidopsis male meiosis [1,19-21]. MMD1/DUET regulates the expression of meiotic genes, including TDM1, JASON, and CAP-D,3, through binding to the H3K4me2/3 sites in the promoter regions [22,23]. The null mmd1/duet mutant displays chromosome de-condensation at metaphase I, altered meiosis progression, irregular spindle organization at anaphase II, and aberrant phragmoplast formation at telophase II, which lead to meiocyte cell death, meiotic restitution, and impaired plant fertility [22-25].

Table 1. Genes, dysfunction of which may cause male meiotic restitution in Arabidopsis thaliana.

\begin{tabular}{|c|c|c|}
\hline Genes & Gene Function & Reference \\
\hline AESP & Proper disjunction of chromosomes & (Liu and Makaroff, 2006) [26] \\
\hline AFH14 & Organization of microtubule and microfilament arrays & (Li et al., 2010) [27] \\
\hline ANQ1/MKK6 & Male meiotic cytokinesis & (Takahashi et al., 2010) [16] \\
\hline CALS5 & Callose synthase & (Dong et al., 2005) [28] \\
\hline CDM1 & Callose metabolism & (Lu et al., 2014) [29] \\
\hline CYCA1;2/TAM & Meiosis II cell cycle transition & (d'Erfurth et al., 2010) [11] \\
\hline MMD1/DUET & $\begin{array}{l}\text { Chromatin condensation, spindle and phragmoplast } \\
\text { formation }\end{array}$ & (Andreuzza et al., 2015; Wang et al., 2016) [22,23] \\
\hline DYAD/SWITCH1 & Meiotic chromosome organization & (Ravi et al., 2008) [30] \\
\hline GSL1 and 5 & Callose wall formation & (Enns et al., 2005) [31] \\
\hline JASON & Meiosis II spindle orientation & $\begin{array}{l}\text { (Brownfield et al., 2015; De Storme and Geelen, } \\
\text { 2011) }[13,32]\end{array}$ \\
\hline KINESIN-12 & Polymerization of phragmoplast microtubules & (Liu et al., 2010; Oh et al., 2014) $[33,34]$ \\
\hline MPK4 & Male meiotic cytokinesis & (Kosetsu et al., 2010; Zeng et al., 2011) [18,35] \\
\hline MS4 & Meiosis progression & (Chaudhury et al., 1994) [36] \\
\hline MYB33/65 & GA signaling downstream transcription regulators & (Liu et al., 2017; Millar and Gubler, 2005) [37,38] \\
\hline OSD1 & Meiosis cycle transition & (Cromer et al., 2012; d'Erfurth et al., 2009) [19,39] \\
\hline PANS1 & $\begin{array}{l}\text { Centromeric cohesion maintenance of sister chromatid in } \\
\text { meiosis II }\end{array}$ & (Zamariola et al., 2014) [40] \\
\hline AtPS1 & Meiosis II spindle orientation & (Brownfield et al., 2015; d'Erfurth et al., 2008) [12,32] \\
\hline$R G A$ and $G A I$ & GA signaling repressors & (Liu et al., 2017) [37] \\
\hline RSW4 & RMA organization & (Yang et al., 2011) [41] \\
\hline SDS & Homolog synapsis and recombination & (Azumi et al., 2002) [20] \\
\hline SHUGOSHIN $1 / 2$ & Centromeric cohesion maintenance at anaphase I & (Zamariola et al., 2013; Zamariola et al., 2014) $[40,42]$ \\
\hline TES2 & Male meiotic cytokinesis & (Spielman et al., 1997; Yang et al., 2003a) $[15,17]$ \\
\hline TIO & Male meiotic cytokinesis & (Oh et al., 2014) [34] \\
\hline
\end{tabular}

In search of genetic factors that contribute to ploidy consistency in meiosis, we previously performed a forward genetic screen of ethyl methanesulfonate (EMS)-mutagenized Arabidopsis thaliana Col-0 plants for mutants that produce over-sized pollen grains [13]. Here, the mutant male meiotic restitution 1 ( $\mathrm{mmr} 1$ ) is described to consistently produce a relatively small fraction of unreduced pollen grains. The mmr1 mutant undergoes meiotic restitution and produces diploid and/or polyploid microspores. Meanwhile, mmr1 displays a mildly impacted chromosome condensation at metaphase I. Furthermore, the organization of spindles and phragmoplast in late meiosis II meiocytes of $m m r 1$ are interfered, which results in defective cytokinesis. The causative point mutation in mmr1 was mapped to the third exon of MMD1/DUET within the conserved PHD domain. Overall, we here describe an allelic and hypomorphic mutant of Arabidopsis chromatin regulator MMD1/DUET. 


\section{Materials and Methods}

\subsection{Plant Materials and Growth Conditions}

Arabidopsis thaliana wild-type accession Col-0 was used in this study. EMS-mutagenized Col-0 seeds were obtained from the Nottingham Arabidopsis Stock Centre. Arabidopsis $p W O X 2:: C E N H 3-G F P$ [43] and $m m d 1 /$ duet [22] were previously described. Seeds were germinated on $\mathrm{K} 1$ medium for 6 to 8 days, and seedlings were transferred to soil and cultivated in growth chambers at $12 \mathrm{~h}$ light $/ 12 \mathrm{~h}$ night, $20^{\circ} \mathrm{C}$, and $70 \%$ humidity. Upon bolting, the photoperiod was changed to a $16 \mathrm{~h}$ day $/ 8 \mathrm{~h}$ night regime.

\subsection{Cytology and Microscopy}

Pollen 4',6-diamidino-2-phenylindole (DAPI) staining, callosic cell wall staining, and analysis of the male meiotic products (tetrad-stage analysis by orcein and DAPI staining) were performed as described [21]. Sperm formation and chromosome counting was performed using the fluorescent marker $p W O X 2:: C E N H 3-G F P$ and $p M G H 3:: H 2 B-G F P$ lines. Spores were released in a $0.05 \mathrm{M}$ phosphate buffer ( $\mathrm{pH} 7.0$ ) containing $0.5 \%$ Triton X-100 $(v / v)$. Meiotic chromosomes were visualized following meiotic chromosome spreading and microtubules using immunostaining as described [21,44]. The assessment of unreduced pollen and microspores was performed by comparing the size to the haploid pollen (diameter $>28 \mu \mathrm{m}$ ) or by counting the number of nuclei [45]. Bright-field and fluorescence microscopy were performed using an Olympus IX81 inverted fluorescence microscope equipped with an X-Cite Series 120Q UV lamp and an Olympus XM10 camera. Bifluorescent images and Z-stacks were processed using ImageJ. Brightness and contrast settings were adjusted using Photoshop CS6.

\subsection{Identification of the Causative Mutation}

The causative mutation in $m m r 1$ was identified through bulk segregate analysis of progeny from a Col-0/Ler hybrid. The microsporogenesis of the F1 progenies obtained by homozygous $m m r 1$ plants (female) crossing with Ler plants (male) were checked under microscope, and the genotype of the F1 progenies was examined by PCR using the primers listed in Supplementary Table S1. F2 population was collected by F1 selfing. Then, 1100 F2 individuals were checked and the samples showing meiotic restitution/samples phenocopied wild-type plants $=1: 3$, confirming that the meiotic restitution in $m m r 1$ is caused by a single recessive mutation. F2 plants showing more than $5 \%$ oversized pollen grains were selected and genomic DNA was isolated and pooled for sequencing.

\section{Results}

\subsection{Isolation of mmr1 That Produces Diploid and/or Polyploid Pollen Grains}

Previously, we screened an M2 Col-0 Arabidopsis EMS-mutagenized population for mutants that produce pollen grains with enlarged sizes. In this screen, we identified the male meiotic restitution 1 ( $m m r 1$ ) that produces approximately $7.1 \%$ over-sized pollen grains (Figure 1A,C, Col-0 and D, mmr1). 4',6-diamidino-2-phenylindole (DAPI) staining revealed that the enlarged pollen contained larger sperms indicative of a higher DNA content (Figure 1E, Col-0; F, mmr1; Supplementary Figure S1A-C). In support, mmr1 plants expressing $p M G H 3:: H 2 B-G F P$ that labels sperm nuclei [46] exhibited larger H2B fluorescent signals (Figure 1G, Col-0; H, mmr1; Supplementary Figure S1D-F). Occasionally, the enlarged $m m r 1$ pollen grains were found to contain more than two sperm (Supplementary Figure S1C,F), indicating a potential defect in cell division or chromosome dynamics. Unicellular stage microspores were next examined, which showed that $m m r 1$ generated larger microspores with multiple nuclei (Figure 1I,J, Col-0; K-O, mmr1), suggesting that the enlarged pollen grains originated from the over-sized microspores. We then introduced $p$ WOX2::CENH3-GFP into the mmr1 mutant to determine the exact chromosome number of the enlarged gametes [43]. Most unicellular stage microspores from wild-type showed five $\mathrm{CENH} 3$ foci, which represent halved chromosome counts (Figure 1B,P). In contrast, the enlarged mmr1 microspores contained either $10(12.8 \%)$ or $15(1.1 \%)$ CENH3 
foci (Figure 1B,Q; Supplementary Figure S2A,B), indicating these over-sized microspores were diploid and/or triploid, respectively. Meanwhile, the $m m r 1$ mutant showed normal silique development (Supplementary Figure S3A,B) but yielded less seeds than wild-type plants (Supplementary Figure S3C). These findings suggest that $m m r 1$ produces unreduced gametes and has an interfered fertility.

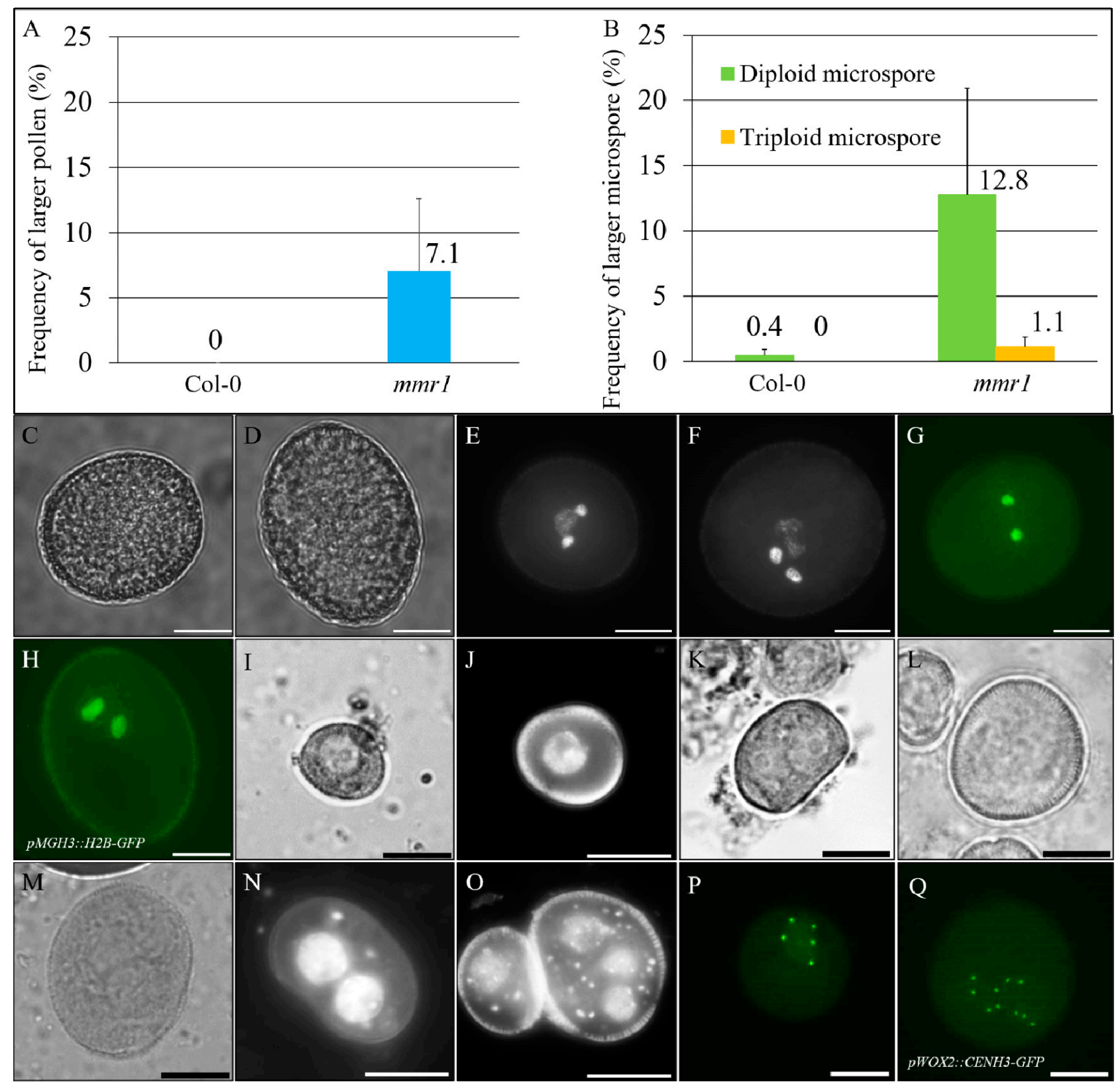

Figure 1. Male meiotic restitution 1 ( $m m r 1)$ generates unreduced gametes. (A,B) Histograms showing the frequency of enlarged pollen grains (A) and diploid and/or triploid unicellular stage microspores (B) in $m m r 1$. Error bars represent standard deviation of the mean values. Numbers indicate the average rate of the corresponding phenotypes. Three bioreplicates were analyzed. (C,D) Bright field images of mature pollen grains in Col-0 (C) and mmr1 (D). (E,F) DAPI-stained mature pollen grains in Col-0 (E) and $m m r 1(\mathbf{F}) .(\mathbf{G}, \mathbf{H})$ Mature pollen grains expressing pMGH3::H2B-GFP in Col-0 (G) and mmr1 (H). (I,J) Unicellular stage microspores by bright field imaging (I) and DAPI-staining (J) in Col-0. (K-O) Unicellular stage microspores by bright field imaging (K, diploid; L, triploid; $\mathbf{M}$, tetraploid) and DAPI staining (N, diploid; O, triploid) in mmr1. (P,Q) Unicellular stage microspores expressing $p W O X 2:: C E N H 3-G F P$ in Col-0 (P) and $m m r 1(\mathbf{Q})$. Scale bars $=10 \mu \mathrm{m}$. 


\section{2. mmr1 Undergoes Meiotic Restitution}

The formation of unreduced microspores suggests that meiotic restitution occurred in the microsporogenesis of $m m r 1$. We hence analyzed and quantified tetrad-stage meiocytes in $m m r 1$ using orcein staining. Wild-type plants consistently generated balanced tetrads that contained four haploid spores with each harboring one nucleus (Figure 2A,B). However, $m m r 1$ plants were found to produce approximately $35.0 \%$ meiotic restituted products (Figure 2A) with 19.9\% triads (Figure 2A,E), 8.7\% unbalanced dyads (Figure 2A,D), 4.6\% balanced dyads (Figure 2A,C) and 1.9\% monads (Figure 2A,F), respectively. DAPI staining of tetrad-stage meiocytes confirmed the formation of dyad and triad with more than one nucleus per spore, which represented occurrence of meiotic restitution in the mmr1 mutant (Figure 2G, Col-0; H,I, mmr1).
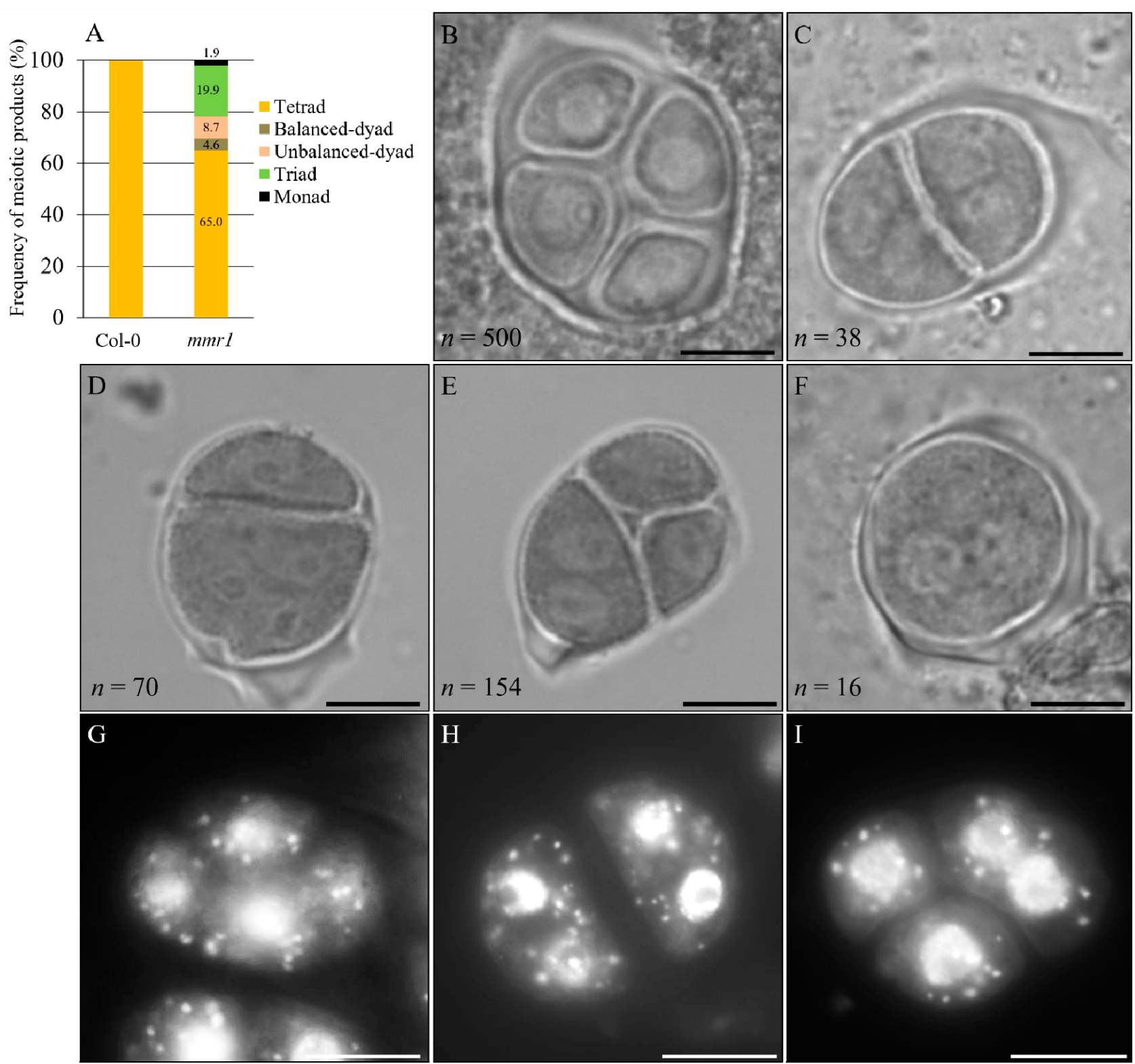

Figure 2. $m m r 1$ undergoes meiotic restitution. (A). Histogram showing the frequency of tetrad-stage meiotic products generated by mmr1 plants. Numbers indicate the frequency of the meiotic products. (B) Normal tetrad in Col-0. (C-F) Balanced dyad (C), unbalanced dyad (D), triad (E), and monad (F) in $m m r 1 . n$ represents the number of cells used for quantification, and five biological individuals were analyzed. (G-I) DAPI-stained tetrad stage meiocytes in Col-0 (G) and $m m r 1$ (H,I). Scale bars $=10 \mu \mathrm{m}$. 
Meiotic spreading was performed to monitor the chromosome behaviors in $m m r 1$ meiosis (Figure 3). In wild-type plants, homologous chromosomes were fully paired at pachytene (Figure 3A), and five pairs of bivalents occurred at diakinesis (Figure 3B). No obvious defect was observed in $m m r 1$ meiocytes at these stages, indicating normal homolog synapsis and crossover formation (Figure 3I,J). At metaphase I, most bivalents in the wild-type showed chromosomes under tension (ratio of bivalents with tension/bivalents without tension $=2.5$ ), aligned at the equatorial plate (Figure 3C). In $m m r 1$, however, more bivalents displayed an aberrant shape at metaphase I and lacked the typical thin threads at either side that result from the pulling force at the kinetochore (bivalents with tension/bivalents without tension $=1.5$ ) (Figure 3K). The minor difference of metaphase I chromosomes suggested that the mmr1 mutation has a mild impact on chromosome condensation. $m m r 1$ chromosomes behaved normally from the interkinesis to metaphase II stages as control (Figure 3D,E, Col-0; L,M, mmr1). At anaphase II, the sister chromatids in $m m r 1$ separated, indicating regular sister chromatid cohesion dynamics (Figure 3F, Col-0; N, mmr1). At telophase II, however, unlike Col-0 meiocytes that displayed four balanced isolation of chromosome sets, $m m r 1$ occasionally showed clustered chromosomes representing restitution configurations (Figure $3 \mathrm{G}, \mathrm{Col}-0 ; \mathrm{O}, m m r 1$ ), which consequently led to adjacent or fused nuclei at tetrad stage (Figure $3 \mathrm{H}, \mathrm{Col}-0$; P, mmr1; Supplementary Figure S4A-C). These data indicate that although both homologous chromosomes and sister chromatids in the $m m r 1$ mutant were able to segregate, positioning of haploid chromosome sets at telophase II was somehow interfered, which implied an alteration in proper spindle orientation and/or cytokinesis.

\subsection{Meiotic Cytokinesis Is Defective in mmr1}

To determine whether meiotic restitution in $m m r 1$ was caused by a defect in cytokinesis, we analyzed meiotic cell walls using aniline blue staining that specifically marks callose. Tetrads in the wild-type plants generated a cross-shaped cell wall configuration, indicating four haploid spores (Figure $4 \mathrm{~A}$ ). In contrast, $m m r 1$ meiocytes were observed to have one or more cell walls omitted, displaying balanced dyads (Figure 4B), unbalanced dyads (Figure 4C,E) or triads (Figure 4D,F). Moreover, interrupted cell walls were occasionally observed (Figure $4 \mathrm{E}, \mathrm{F}$, see red arrows). These figures indicate that $m m r 1$ is defective for generating complete meiotic cell walls during cytokinesis. 


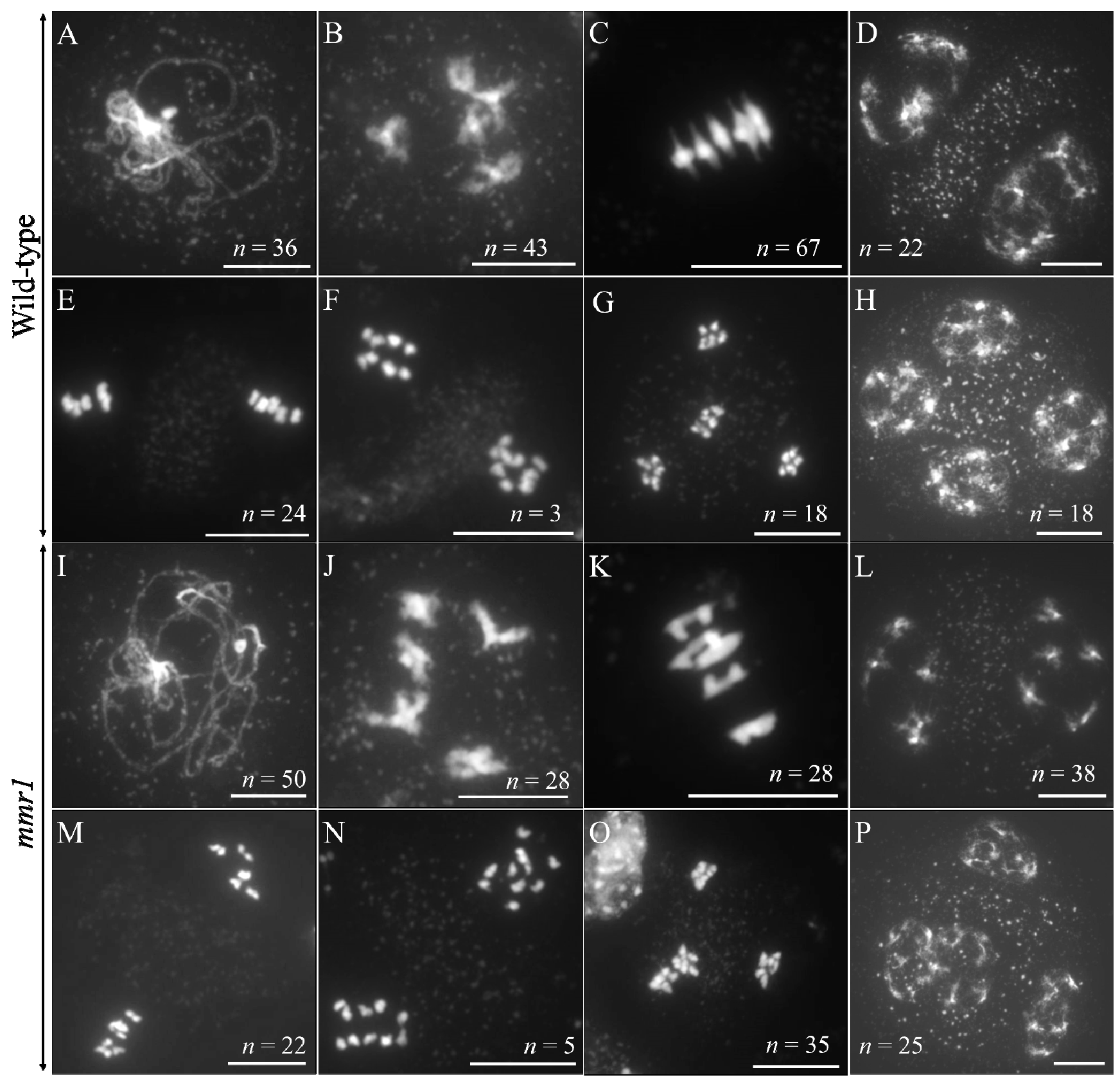

Figure 3. Meiotic spreading of wild-type Col-0 and mmr1 plants. (A-H) DAPI-stained chromosomes at pachytene (A), diakinesis (B), metaphase I (C), interkinesis (D), metaphase II (E), anaphase II (F), telophase II (G), and tetrad stage (H) in Col-0. (I-P) DAPI-stained chromosomes at pachytene (I), diakinesis (J), metaphase I (K), interkinesis (L), metaphase II (M), anaphase II (N), telophase II (O), and tetrad stage (P) in $m m r 1$. $n$ indicates the number of observed cells at corresponding stages. Scale bars $=10 \mu \mathrm{m}$. 

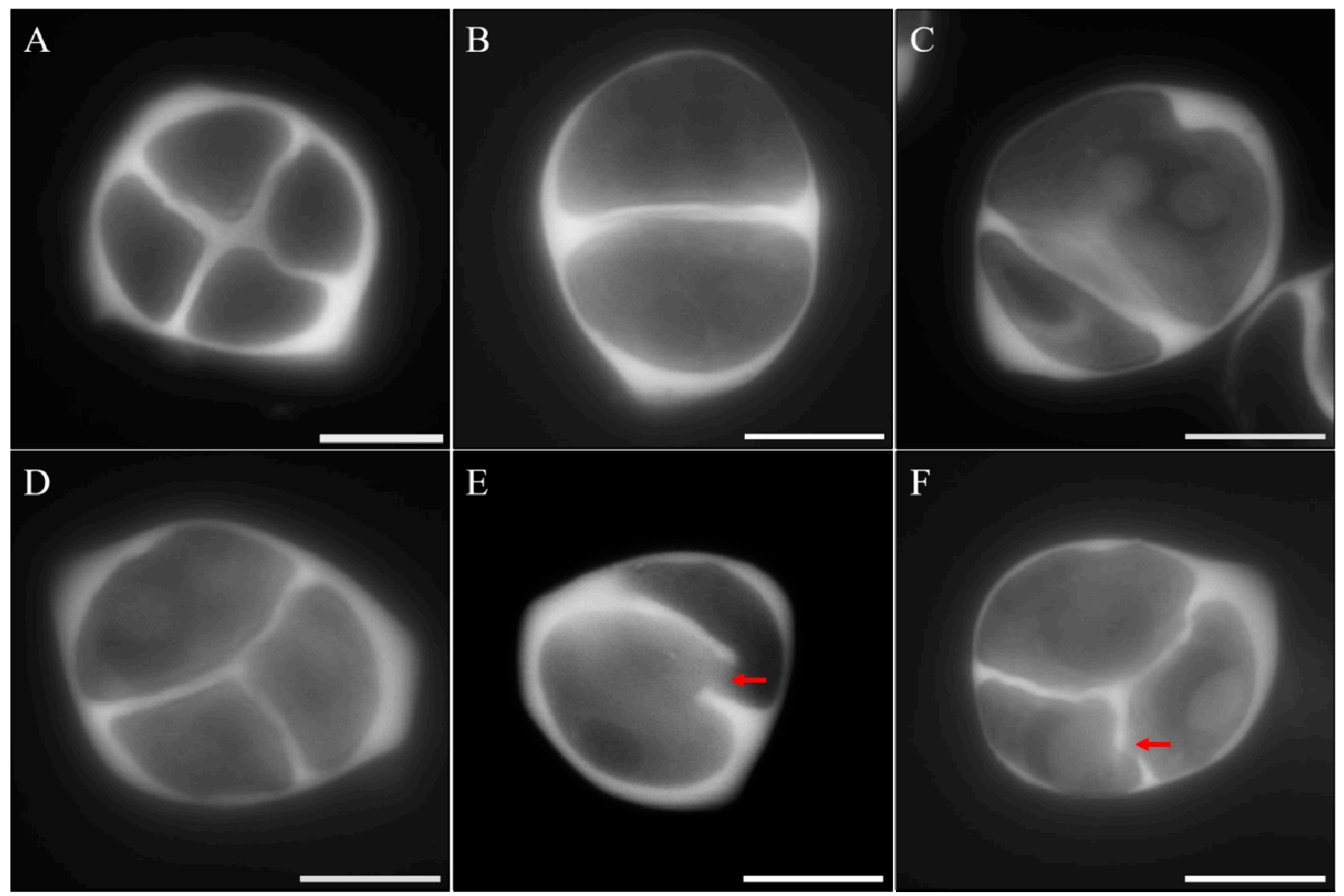

Figure 4. Meiotic cell formation is defective in mmr1. (A) Tetrad with a complete meiotic cell wall in the wild-type. (B-F) Balanced dyad (B), unbalanced dyads (C,E), and triads (D and F) in mmr1. Red arrows indicate the interruptions in meiotic cell walls of $m m r 1$. Scale bars $=10 \mu \mathrm{m}$.

\subsection{Altered Spindle Orientation and Phragmoplast Formation in mmr1}

Construction of meiotic cell walls relies on the organization of the microtubular cytoskeleton surrounding chromosomes or nuclei [21]. Immunostaining of $\alpha$-tubulin was therefore applied to elucidate whether the meiotic cytokinesis defects in $m m r 1$ was caused by any alteration in microtubules. From prophase I to metaphase II, $m m r 1$ did not show any obvious difference in microtubular network formation, as in wild-type plants (Figure 5B,D,F,H,J, Col-0; C,E,G,I,K, mmr1). Successfully generated spindles at metaphase I and II supported the observation that both chromosome segregation cycles were not influenced in $m m r 1$ (Figure 5E,K). However, at telophase II, the orientation of phragmoplast in $m m r 1$ displayed either parallel or tripolar configuration (Figure 5L, Col0; M,N, mmr1; Supplementary Figure S5A,B). In wild-type and most mmr1 tetrad stage meiocytes, radial microtubule arrays (RMAs) were organized into mini-phragmoplasts between the separated nuclei, contributing to tetrad formation (Figure 5O, Col-0; P, mmr1). Approximately $48.0 \%$ tetrad stage meiocytes in $m m r 1$, however, showed omission of one or more RMAs between the separated nuclei (Figure 5A; Supplementary Table S2), which generated triads (36.3\%) (Figure 5S; Supplementary Figure S5G,H), balanced-dyads (7.8\%) (Figure 5Q; Supplementary Figure S5C,D) and unbalanced dyads (3.9\%) (Figure 5R; Supplementary Figure S5E,F). In addition, the telophase II phragmoplasts in $m m r 1$ were broader than in Col- 0 and contained microtubules that were not in line with the axes across the nuclei (Figure 5L, Col-0; M,N, and Supplementary Figure S5G, mmr1). Meanwhile, the microtubules of RMAs were also less compacted than in Col-0 (Figure 5O, Col-0; P-S and Supplementary Figure $\mathrm{S} 5 \mathrm{C}-\mathrm{H}, \mathrm{mmr} 1)$. Taken together, these findings revealed defective 
spindle orientation, and formation and/or organization of phragmoplasts at meiosis II in the mmr1 mutant.
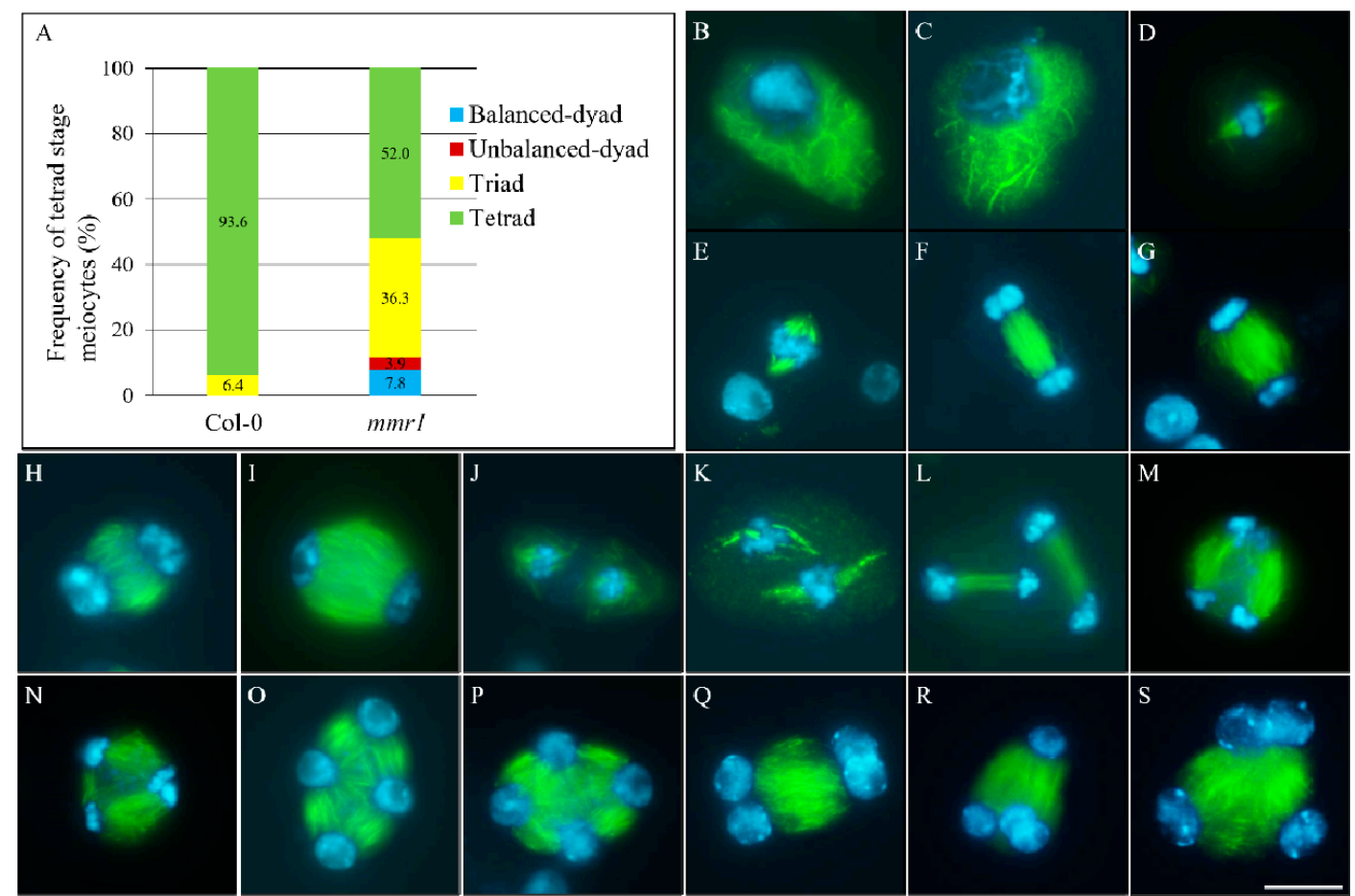

Figure 5. Microtubular cytoskeleton structures in mmr1 meiocytes. (A) Histogram showing the frequency of tetrad stage meiocytes in $m m r 1$. Numbers indicate the frequency of the corresponding phenotypes. (B-S) Prophase I- (B,C), metaphase I- (D,E), anaphase I- (F,G), interkinesis- $(\mathbf{H}, \mathbf{I})$, metaphase II- $(\mathbf{J}, \mathbf{K})$, telophase II- (L-N), and tetrad-stage $(\mathbf{O}-\mathbf{S})$ meiocytes in Col-0 (B,D,F,H,J,L,O) and the $m m r 1$ mutant $(\mathbf{C}, \mathbf{E}, \mathbf{G}, \mathbf{I}, \mathbf{K}, \mathbf{M}, \mathbf{N}, \mathbf{P}-\mathbf{S})$. Green, $\alpha$-tubulin; cyan, DAPI. Scale bar $=10 \mu \mathrm{m}$.

\section{5. mmr1 Carries a Point Mutation in the PHD-Finger Domain of MMD1/DUET}

To identify the causative mutation in the $m m r 1$ mutant, we performed bulk segregant analysis combined with whole genome sequencing. F1 progenies from the intercrossing between $m m r 1$ mutant and wild-type Landsberg erecta (Ler) was genotyped using simple sequence length polymorphism (SSLP) primers to check Col0/Ler heterozygosity (Supplementary Figure S6). All of the F1 individuals displayed normal male meiotic cytokinesis and produced normal-sized male gametes (Supplementary Figure S7A-C), suggesting a recessive mutation in $m m r 1$. The phenotypic screening of $1100 \mathrm{~F} 2 \mathrm{Col} / \mathrm{Ler}$ descendants resulted in 250 individuals producing unreduced microspores, and DNA from individuals were pooled and sequenced. A $26 \mathrm{Mb}$ region on chromosome 1 was enriched for Col-0 SNPs (Supplementary Figure S8). Several genes within this region carried mutations, but the candidate gene MMD1/DUET (AT1G66170) was the only meiosis-specific one. mmr1 was found to carry a single C-to-T transition at the genomic position $2168 \mathrm{bp}$ from the start codon of MMD1/DUET, leading to an amino acid change G618D (Figure 6A; Supplementary Table S3). The G618D amino acid change was located within the plant homeodomain (PHD) of MMD1/DUET, which has been found in many chromatin regulatory factors and conserved in histone binding proteins (Figure 6B) [24]. A complementation test was performed by crossing homozygous mmr 1 mutant with homozygous $m m d 1$ null mutant using mmr1 as the pollen donor. All checked F1 progenies produced unreduced microspores and pollen, and showed defects in meiotic cytokinesis (Figure 6C-G, Col-0; H-O, mmr1/mmd1 $\mathrm{F} 1$ progenies), which indicates that $m m r 1$ is allelic to MMD1/DUET. Since $m m r 1$ produced a small population of unreduced microspores, it is thus a hypomorphic mmd1/duet allele. 


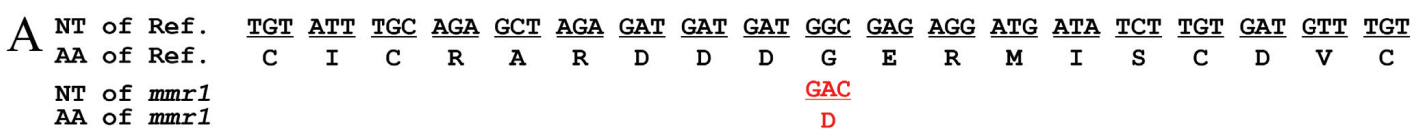

GAG GTA TGG CAA CAT ACA CGA TGT TGT GGG ATT GAT GAT TCA GAT ACT TTG CCT CCA

$\frac{\text { CTT }}{\mathrm{L}} \frac{\text { TTT }}{\mathrm{F}} \frac{\mathrm{GTT}}{\mathrm{V}} \frac{\mathrm{TGT}}{\mathrm{C}} \frac{\mathrm{TCG}}{\mathrm{S}} \frac{\mathrm{AAT}}{\mathrm{N}} \frac{\mathrm{TGC}}{\mathrm{C}} \frac{\mathrm{TGT}}{\mathrm{C}} \frac{\mathrm{GAA}}{\mathrm{E}} \frac{\mathrm{GAA}}{\mathrm{E}}$

В AT1G33420 AT3G05670

AT4G10600

AT5G22260

AT1G66170

conserved. $\operatorname{mm} 1$

DCKCGTKDDDGERMIACDGCGVWHHTRCIGINNADALPSKFLCFRC DCKCGARDDDGERMVACDACKVWHHTLCNS IEDDEAVPSVFLCNMC CTECHOGDDDG-LMLLCDLCDSSAHTYCVGLGRE-VPEGNWYCEGC DCVCGVNFDDGKEMVDCDECGVWVHTWCSRYVKG---DDLFVCHKC ECECGATEEDGERMVCCDICEVWQHTRCVGVQHNEEVPRIFLCQSC KCICRARDDDGERMISCDVCEVWQHTRCCGIDDSDTLPPLFVCSNC ...C.....DG. . M. . CD . C . . . HT . C . . . . . . . . . C . C
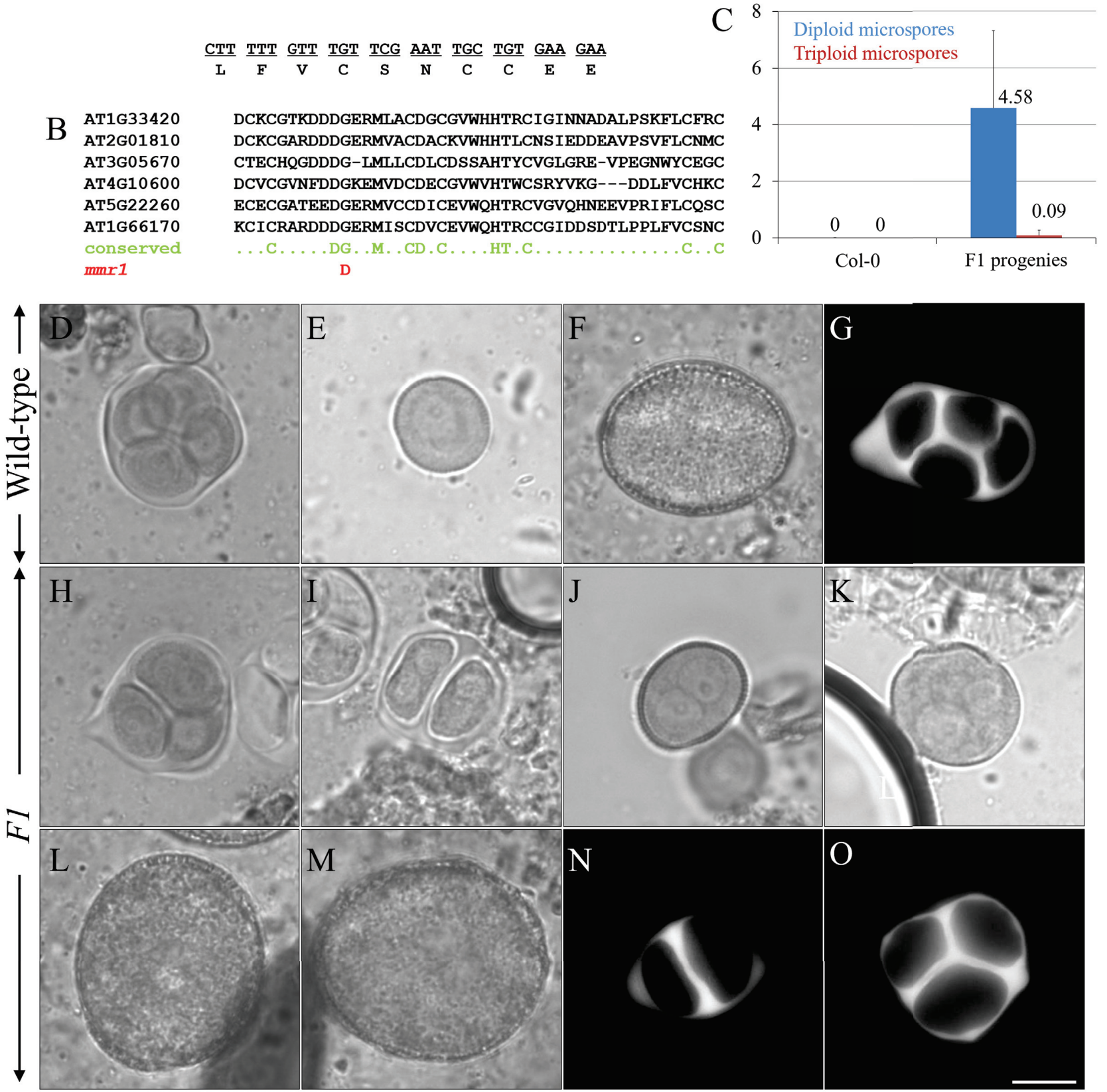

Figure 6. $m m r 1$ is an allelic mutant of MMD1/DUET. (A) Figure showing the nucleotide (NT) sequence and amino acid sequence of the PHD domain in the MMD1/DUET gene and protein, and the changes of that in mmr1. (B) Figure showing the conservation of the amino acid in the PHD domain in multiple histone binding proteins, and the point mutation-caused amino acid replacement in that of $m m r 1$. (C) Histogram showing the frequency of diploid and triploid microspores produced by $m m r 1 / m m d 1$ F1 progenies. Nine individuals were quantified. Numbers indicate the frequency of diploid and/or triploid microspores. (D,H,I) Orcein-stained tetrads in the Col-0 plants (D), and triad (H) and dyad (I) in the $m m r 1 / m m d 1 \mathrm{~F} 1$ progenies. (E,J,K) Haploid unicellular stage microspore in the Col-0 plants (E), and diploid (J) and polyploid (K) unicellularstage microspores in the $m m r 1 / m m d 1 \mathrm{~F} 1$ progenies. (F,L,M) Haploid pollen grain in the Col-0 plants $(\mathbf{F})$, and enlarged pollen grains in the $m m r 1 / m m d 1 \mathrm{~F} 1$ progenies $(\mathbf{L}, \mathbf{M}) .(\mathbf{G}, \mathbf{N}, \mathbf{O})$ Aniline blue-stained meiotic cell walls in the Col-0 plants $(\mathbf{G})$ and in the $m m r 1 / m m d 1$ F1 progenies $(\mathbf{N}, \mathbf{O})$. Scale bar $=10 \mu \mathrm{m}$. 


\section{Discussion}

In this study, we reported the identification of a recessive mutant $m m r 1$ that consistently produces a small fraction (around $5 \%-10 \%$ ) of unreduced pollen grains. The phenotype is caused by a single amino acid change in the PHD domain of MMD1/DUET, a chromatin regulator that is specifically expressed in male meiocytes and functions in male meiosis [24,25]. Ds transposon insertions in the exon of MMD1/DUET lead to MMD1/DUET null mutants, which display strong male sterility due to various defects in meiosis that culminate into meiocyte cell death [24,25]. Male meiosis in MMD1/DUET is initiated normally, forming meiocytes that progress up to pachytene as in the wild-type. During the subsequent steps in the meiosis program, two cycles of chromosome condensation take place. In $m m d 1 / d u e t$, diakinesis chromosomes are paired into bivalents and appear less compact and show inter-bivalent interactions $[24,25,47]$, which were, however, not observed in $m m r 1$. In $m m r 1$, an alteration in chromosome structure was observed at metaphase I, when bivalents are under tension from kinetochore microtubule pulling forces. In the wild-type, the kinetochores are slightly pulled apart, which is evident from the thin DAPIstained threads perpendicular to the equatorial plane. These extensions are also absent, or at least far less pronounced, in null $m m d 1 /$ duet [23]. In $m m r 1$, the altered chromosome condensation in metaphase I is not as severe as in the null $m m d 1 /$ duet mutant, suggesting that $m m r 1$ is hypomorphic. The reduction in fertility of $m m r 1$ is very mild compared to $m m d 1 /$ duet, in line with the occurrence of cytological defects in only a subpopulation of the meiocytes and microspores. The $m m r 1$ mutation therefore has a minor impact on the functioning of $M M D 1 / D U E T$.

The G618D amino acid change in $m m r 1$ is located within the PHD domain of MMD1/DUET, which forms an aromatic cage, required for the specific binding to H3K4me2 [22,23,47]. MMD1/DUET may bind a wider range of histone peptides, as a recombinant peptide encompassing the MMD1/DUET PHD finger (601 to 659) was shown to bind the histone peptides $\mathrm{H} 3 \mathrm{~K} 4 \mathrm{me} 2$ /me3, H3K9me2, and H3S10 [23]. The G618D amino acid change in the $m m r 1$ allele introduces a negative charge in the PHD finger, modifying its electrostatic balance and likely changing the strength and/or specificity of histone binding. As $m m r 1$ displays a hypomorphic phenotype, we speculate that the $m m r 1$ allele has the same histone specificity but with slightly weaker binding affinity. The PHD domain is of critical importance to MMD1/DUET function, as PHD deletion mutants and selected PHD amino acid changes show the same sterility phenotype as a knockout line [23,47]. Andreuzzi et al. (2015) [22] reported on a hypomorphic MMD1/DUET PHD finger mutation that causes the formation of a low frequency of enlarged pollen grains (approximately $5 \%$ ) reminiscent to $m m r 1$ [22]. The reported hypomorphic mutant appeared to be defective specifically in meiosis II spindle and RMA organization [22]. Chromosome condensation, M II phragmoplast, and RMA organization are affected in mmr1, suggesting that the G618D amino acid change interferes with MMD1/DUET functioning during both meiosis I and II. The mild alteration in chromosome condensation at metaphase I in $m m r 1$ suggests that the point mutation G618D is more related to MMD1/DUET function in phragmoplast organization.

\section{Conclusions}

MMD1/DUET has been shown to profoundly modulate gene expression in anthers and seems to do this differentially during early stage and late stage of meiosis [23]. Expression of the meiotic cell cycle regulator TDM and the meiosis spindle II organizer $J A S O N$ is severely reduced in duet, in line with the defects in spindle position and cytokinesis [22]. MMD1/DUET also directly regulates the expression of the condensin genes $C A P-D 2, C A P-D 3, C A P-H$, and $C A P-H 2$. The cap-d3 mutants show a male meiotic chromosome condensation phenotype such as $m m d 1 /$ duet, indicating that the prophase I chromosome condensation phenotype is due the misexpression of CAP-D3 in MMD1/DUET [23]. Since $m m r 1$ shows a very mild phenotype in metaphase I chromosome condensation and a low frequency of meiotic restitution, we speculate that a reduction in the expression of 
CAP-D3 and Jason is minor and only occasionally drops below a threshold to generate the observed defects in chromosome condensation and cytokinesis. The isolated $m m r 1$ mutant in this report thus provides a tool to analyze the function of regions within the PHD domain in MMD1/DUET with a specific correlation to telophase II and cytokinesis.

Supplementary Materials: The following are available online at https:/ / www.mdpi.com/article/ 10.3390/genes12040516/s1, Supplementary Figure S1. mmr1 produces enlarged pollen grains with increased DNA content; Supplementary Figure S2. mmr1 produces diploid or polyploid microspores; Supplementary Figure S3. mmr1 displays a mildly reduced fertility; Supplementary Figure S4. Tetradstage meiocytes in the $m m r 1$ mutant stained by DAPI; Supplementary Figure S5. mmr1 exhibits defective phragmoplasts and radial microtubule array (RMA); Supplementary Figure S6. Genotyping of the F1 individual obtained by crossing between homozygous mmr1 mutant and wild-type Ler plants using simple sequence length polymorphism (SSLP) marker primer F3L12; Supplementary Figure S7. Phenotyping of F1 plants obtained by crossing homozygous mmr1 mutant with wild-type Ler plants; Supplementary Figure S8. Whole genome sequencing analysis of pooled mmr1/Ler F2 plants showing the large pollen phenotype; Supplementary Table S1. Primer for genotyping of F1 progenies ( $m m r 1$ crossing with wild-type Ler); Supplementary Table S2. Quantification of mmr1 meiotic restitution by monitoring microtubules; Supplementary Table S3. Mutation of $m m r 1$ at the MMD1/DUET locus.

Author Contributions: B.L., C.J., N.D.S., S.S., C.S. and T.D.M. performed the experiments. B.L. analyzed the data and wrote the manuscript. N.D.S. and D.G. conceived and designed the research project, analyzed the data, and edited the manuscript. All authors have read and agreed to the published version of the manuscript.

Funding: This work was supported by the National Natural Science Foundation of China (32000245 to B.L.), the Hubei Provincial Natural Science Foundation of China (2020CFB159 to B.L.), Fundamental Research Funds for the Central Universities, South-Central University for Nationalities (CZY20001 to B.L.), the China Scholarship Council (201306760005 to B.L.), the FWO PostDoc grant (1293014N to N.D.S.), and Fundamental Research Funds for the Central Universities, South-Central University for Nationalities (YZZ18007 to B.L.).

Institutional Review Board Statement: Not applicable.

Informed Consent Statement: Not applicable.

Data Availability Statement: No new data were created or analyzed in this study. Data sharing is not applicable to this article.

Acknowledgments: The authors thank Imran Siddiqi for kindly sharing the null mmd1 mutant.

Conflicts of Interest: All authors declare that there are no conflicts of interest for the publication.

\section{References}

1. Del Pozo, J.C.; Ramirez-Parra, E. Whole genome duplications in plants: An overview from Arabidopsis. J. Exp. Bot. 2015, 66, 6991-7003. [CrossRef] [PubMed]

2. Ren, R.; Wang, H.F.; Guo, C.C.; Zhang, N.; Zeng, L.P.; Chen, Y.M.; Ma, H.; Qi, J. Widespread whole genome duplications contribute to genome complexity and species diversity in angiosperms. Mol. Plant 2018, 11, 414-428. [CrossRef] [PubMed]

3. Bretagnolle, F.; Thompson, J.D. Gametes with the somatic chromosome number: Mechanisms of their formation and role in the evolution of autopolyploid plants. New Phytol. 1995, 129, 1-22. [CrossRef]

4. Ramsey, J.; Schemske, D.W. Pathways, mechanisms, and rates of polyploid formation in flowering plants. Annu. Rev. Ecol. Syst. 1998, 29, 467-501. [CrossRef]

5. Brownfield, L.; Kohler, C. Unreduced gamete formation in plants: Mechanisms and prospects. J. Exp. Bot. 2011, 62, 1659-1668. [CrossRef]

6. De Storme, N.; Geelen, D. Sexual polyploidization in plants-cytological mechanisms and molecular regulation. New Phytol. 2013, 198, 670-684. [CrossRef]

7. De Storme, N.; Geelen, D. Induction and characterization of diploid pollen grains in Arabidopsis thaliana. In Plant Meiosis: Methods and Protocols; Pradillo, M., Heckmann, S., Eds.; Springer: New York, NY, USA, 2020; pp. 331-346.

8. Kreiner, J.M.; Kron, P.; Husband, B.C. Evolutionary dynamics of unreduced gametes. Trends Genet. 2017, 33, 583-593. [CrossRef]

9. Loginova, D.B.; Silkova, O.G. Mechanisms of Unreduced Gamete Formation in Flowering Plants. Russ. J. Genet. 2017, 53, 741-756. [CrossRef]

10. Mason, A.S.; Pires, J.C. Unreduced gametes: Meiotic mishap or evolutionary mechanism? Trends Genet. 2015, 31, 5-10. [CrossRef] 
11. d'Erfurth, I.; Cromer, L.; Jolivet, S.; Girard, C.; Horlow, C.; Sun, Y.; To, J.P.C.; Berchowitz, L.E.; Copenhaver, G.P.; Mercier, R. The cyclin-A CYCA1;2/TAM is required for the meiosis I to meiosis II transition and cooperates with OSD1 for the prophase to first meiotic division transition. PLoS Genet. 2010, 6, e1000989. [CrossRef]

12. d'Erfurth, I.; Jolivet, S.; Froger, N.; Catrice, O.; Novatchkova, M.; Simon, M.; Jenczewski, E.; Mercier, R. Mutations in AtPS1 (Arabidopsis thaliana Parallel Spindle 1) Lead to the Production of Diploid Pollen Grains. PLoS Genet. 2008, 4, e1000274. [CrossRef] [PubMed]

13. De Storme, N.; Geelen, D. The Arabidopsis mutant jason produces unreduced first division restitution male gametes through a parallel/fused spindle mechanism in meiosis II. Plant Physiol. 2011, 155, 1403-1415. [CrossRef] [PubMed]

14. Hülskamp, M.; Parekh, N.S.; Grini, P.; Schneitz, K.; Zimmermann, I.; Lolle, S.J.; Pruitt, R.E. The STUD gene is required for male-specific cytokinesis after telophase II of meiosis in Arabidopsis thaliana. Dev. Biol. 1997, 187, 114-124. [CrossRef]

15. Spielman, M.; Preuss, D.; Li, F.L.; Browne, W.E.; Scott, R.J.; Dickinson, H.G. TETRASPORE is required for male meiotic cytokinesis in Arabidopsis thaliana. Development 1997, 124, 2645-2657. [PubMed]

16. Takahashi, Y.; Soyano, T.; Kosetsu, K.; Sasabe, M.; Machida, Y. HINKEL kinesin, ANP MAPKKKs and MKK6/ANQ MAPKK, which phosphorylates and activates MPK4 MAPK, constitute a pathway that is required for cytokinesis in Arabidopsis thaliana. Plant Cell Physiol. 2010, 51, 1766-1776. [CrossRef] [PubMed]

17. Yang, C.Y.; Spielman, M.; Coles, J.P.; Li, Y.; Ghelani, S.; Bourdon, V.; Brown, R.C.; Lemmon, B.E.; Scott, R.J.; Dickinson, H.G. TETRASPORE encodes a kinesin required for male meiotic cytokinesis in Arabidopsis. Plant J. 2003, 34, 229-240. [CrossRef]

18. Zeng, Q.N.; Chen, J.G.; Ellis, B.E. AtMPK4 is required for male-specific meiotic cytokinesis in Arabidopsis. Plant J. 2011, 67, 895-906. [CrossRef] [PubMed]

19. Cromer, L.; Heyman, J.; Touati, S.; Harashima, H.; Araou, E.; Girard, C.; Horlow, C.; Wassmann, K.; Schnittger, A.; De Veylder, L.; et al. OSD1 promotes meiotic progression via APC/C inhibition and forms a regulatory network with TDM and CYCA1;2/TAM. PLoS Genet. 2012, 8, e1002865. [CrossRef]

20. Azumi, Y.; Liu, D.; Zhao, D.; Li, W.; Wang, G.; Hu, Y.; Ma, H. Homolog interaction during meiotic prophase I in Arabidopsis requires the SOLO DANCERS gene encoding a novel cyclin-like protein. EMBO J. 2002, 21, 3081-3095. [CrossRef] [PubMed]

21. De Storme, N.; Copenhaver, G.P.; Geelen, D. Production of diploid male gametes in Arabidopsis by cold-induced destabilization of postmeiotic radial microtubule arrays. Plant Physiol. 2012, 160, 1808-1826. [CrossRef]

22. Andreuzza, S.; Nishal, B.; Singh, A.; Siddiqi, I. The chromatin protein DUET/MMD1 controls expression of the meiotic gene TDM1 during male meiosis in Arabidopsis. PLoS Genet. 2015, 11, e1005396. [CrossRef]

23. Wang, J.; Niu, B.; Huang, J.; Wang, H.; Yang, X.; Dong, A.; Makaroff, C.; Ma, H.; Wang, Y. The PHD Finger Protein MMD1/DUET Ensures the Progression of Male Meiotic Chromosome Condensation and Directly Regulates the Expression of the Condensin Gene CAP-D3. Plant Cell 2016, 28, 1894-1909. [CrossRef]

24. Reddy, T.V.; Kaur, J.; Agashe, B.; Sundaresan, V.; Siddiqi, I. The DUET gene is necessary for chromosome organization and progression during male meiosis in Arabidopsis and encodes a PHD finger protein. Development 2003, 130, 5975-5987. [CrossRef]

25. Yang, X.; Makaroff, C.A.; Ma, H. The Arabidopsis MALE MEIOCYTE DEATH1 gene encodes a PHD-finger protein that is required for male meiosis. Plant Cell 2003, 15, 1281-1295. [CrossRef]

26. Liu, Z.; Makaroff, C.A. Arabidopsis separase AESP is essential for embryo development and the release of cohesin during meiosis. Plant Cell 2006, 18, 1213-1225. [CrossRef] [PubMed]

27. Li, Y.; Shen, Y.; Cai, C.; Zhong, C.; Zhu, L.; Yuan, M.; Ren, H. The type II Arabidopsis formin14 interacts with microtubules and microfilaments to regulate cell division. Plant Cell 2010, 22, 2710-2726. [CrossRef] [PubMed]

28. Dong, X.; Hong, Z.; Sivaramakrishnan, M.; Mahfouz, M.; Verma, D.P.S. Callose synthase (CalS5) is required for exine formation during microgametogenesis and for pollen viability in Arabidopsis. Plant J. 2005, 42, 315-328. [CrossRef] [PubMed]

29. Lu, P.; Chai, M.; Yang, J.; Ning, G.; Wang, G.; Ma, H. The Arabidopsis CALLOSE DEFECTIVE MICROSPORE1 gene is required for male fertility through regulating callose metabolism during microsporogenesis. Plant Physiol. 2014, 164, 1893-1904. [CrossRef]

30. Ravi, M.; Marimuthu, M.P.A.; Siddiqi, I. Gamete formation without meiosis in Arabidopsis. Nature 2008, 451, 1121. [CrossRef]

31. Enns, L.C.; Kanaoka, M.M.; Torii, K.U.; Comai, L.; Okada, K.; Cleland, R.E. Two callose synthases, GSL1 and GSL5, play an essential and redundant role in plant and pollen development and in fertility. Plant Mol. Biol. 2005, 58, 333-349. [CrossRef]

32. Brownfield, L.; Yi, J.; Jiang, H.; Minina, E.; Twell, D.; Köhler, C. Organelles maintain spindle position in plant meiosis. Nat. Commun. 2015, 6, 6492. [CrossRef] [PubMed]

33. Liu, M.; Nadar, V.C.; Kozielski, F.; Kozlowska, M.; Yu, W.; Baas, P.W. Kinesin-12, a Mitotic Microtubule-Associated Motor Protein, Impacts Axonal Growth, Navigation, and Branching. J. Neurosci. 2010, 30, 14896-14906. [CrossRef] [PubMed]

34. Oh, S.; Bourdon, V.; Dickinson, H.; Twell, D.; Park, S. Arabidopsis Fused kinase TWO-IN-ONE dominantly inhibits male meiotic cytokinesis. Plant Reprod. 2014, 27, 7-17. [CrossRef] [PubMed]

35. Kosetsu, K.; Matsunaga, S.; Nakagami, H.; Colcombet, J.; Sasabe, M.; Soyano, T.; Takahashi, Y.; Hirt, H.; Machida, Y. The MAP kinase MPK4 is required for cytokinesis in Arabidopsis thaliana. Plant Cell 2010, 22, 3778-3790. [CrossRef]

36. Chaudhury, A.; Craig, S.; Dennis, E.; Lavithis, M.; Taylor, P.; Singh, M.; Knox, R.; Signer, E. Genetic control of male fertility in Arabidopsis thaliana: Structural analysis of premeiotic developmental mutants. Sex. Plant Reprod. 1994, 7, 17-28. [CrossRef]

37. Liu, B.; De Storme, N.; Geelen, D. Gibberellin induces diploid pollen formation by interfering with meiotic cytokinesis. Plant Physiol. 2017, 173, 338-353. [CrossRef] 
38. Millar, A.A.; Gubler, F. The Arabidopsis GAMYB-like genes, MYB33 and MYB65, are microRNA-regulated genes that redundantly facilitate anther development. Plant Cell 2005, 17, 705-721. [CrossRef]

39. d'Erfurth, I.; Jolivet, S.; Froger, N.; Catrice, O.; Novatchkova, M.; Mercier, R. Turning Meiosis into Mitosis. PLoS Biol. 2009, 7, e1000124. [CrossRef] [PubMed]

40. Zamariola, L.; De Storme, N.; Vannerum, K.; Vandepoele, K.; Armstrong, S.J.; Franklin, F.C.H.; Geelen, D. SHUGOSHINs and PATRONUS protect meiotic centromere cohesion in Arabidopsis thaliana. Plant J. 2014, 77, 782-794. [CrossRef]

41. Yang, X.; Boateng, K.A.; Yuan, L.; Wu, S.; Baskin, T.I.; Makaroff, C.A. The Radially Swollen 4 separase mutation of Arabidopsis thaliana blocks chromosome disjunction and disrupts the radial microtubule system in meiocytes. PLoS ONE 2011, 6, e19459. [CrossRef]

42. Zamariola, L.; De Storme, N.; Tiang, C.; Armstrong, S.J.; Franklin, F.C.H.; Geelen, D. SGO1 but not SGO2 is required for maintenance of centromere cohesion in Arabidopsis thaliana meiosis. Plant Reprod. 2013, 26, 197-208. [CrossRef] [PubMed]

43. De Storme, N.; Keceli, B.N.; Zamariola, L.; Angenon, G.; Geelen, D. CENH3-GFP: A visual marker for gametophytic and somatic ploidy determination in Arabidopsis thaliana. BMC Plant Biol. 2016, 16, 1. [CrossRef] [PubMed]

44. Liu, B.; De Storme, N.; Geelen, D. Cold-induced male meiotic restitution in Arabidopsis thaliana is not mediated by GA-DELLA signaling. Front. Plant Sci. 2018, 9, 91. [CrossRef] [PubMed]

45. De Storme, N.; Zamariola, L.; Mau, M.; Sharbel, T.F.; Geelen, D. Volume-based pollen size analysis: An advanced method to assess somatic and gametophytic ploidy in flowering plants. Plant Reprod. 2013, 26, 65-81. [CrossRef]

46. Brownfield, L.; Hafidh, S.; Borg, M.; Sidorova, A.; Mori, T.; Twell, D. A plant germline-specific integrator of sperm specification and cell cycle progression. PLoS Genet. 2009, 5, e1000430. [CrossRef]

47. Wang, J.; Yu, C.; Zhang, S.; Ye, J.; Dai, H.; Wang, H.; Huang, J.; Cao, X.; Ma, J.; Ma, H.; et al. Cell-type-dependent histone demethylase specificity promotes meiotic chromosome condensation in Arabidopsis. Nat. Plants 2020, 6, 823-837. [CrossRef] 